\title{
Proper gap-labellings: on the edge and vertex variants*
}

\author{
Celso A. Weffort-Santos ${ }^{1}$, \\ Christiane N. Campos ${ }^{1}$ (Supervisor), Rafael C. S. Schouery ${ }^{1}$ (Co-supervisor) \\ ${ }^{1}$ Institute of Computing - University of Campinas (UNICAMP) \\ Av. Albert Einstein, 1251 - 13083-852 - Campinas - SP — Brazil \\ \{celso.santos, campos, rafael\}@ic.unicamp.br
}

\begin{abstract}
Given a simple graph $G$, an ordered pair $\left(\pi, c_{\pi}\right)$ is said to be a gap$[k]$-edge-labelling (resp. gap-[k]-vertex-labelling) of $G$ if $\pi$ is an edge-labelling (vertex-labelling) on the set $\{1, \ldots, k\}$, and $c_{\pi}$ is a proper vertex-colouring such that every vertex of degree at least two has its colour induced by the largest difference among the labels of its incident edges (neighbours). The decision problems associated with these labellings are NP-complete for $k \geq 3$, and even when $k=2$ for some classes of graphs. This thesis presents a study of the computational complexity of these problems, structural properties for certain families of graphs and several labelling algorithms and techniques. First, we present an NP-completeness result for the family of subcubic bipartite graphs. Second, we present polynomial-time algorithms for families of graphs. Third, we introduce a new parameter associated with gap-[k]-vertex-labellings of graphs.
\end{abstract}

\section{Introduction}

Graph Theory is a field of research within Mathematics which studies graphs using a variety of approaches, including structural and algorithmic. A great number of hard problems, such as timetable or logistic scheduling, pattern matching and register allocation, can be modeled using graphs, particularly as graph colouring problems. The objective in these problems is to assign colours to the elements of the graph subject to some restrictions, e.g. adjacent vertices should receive distinct colours.

Over the past fifty years, generalizations of graph colourings, known as labellings, have been widely studied, due to their rich mathematical aspects. In a labelling of a graph, we assign numeric values (labels) to vertices and/or edges in order to satisfy restrictions imposed by some mathematical function over the set of labelled elements. Many different types of labellings have been studied since the 1960's, to when most authors trace the origin of these types of problems.

Since there are so many different types of graph labellings, it is not surprising that particular problems have gone unnoticed in the literature, and yet pose interesting challenges to the scientific community. While some labellings model real world problems directly, such as vertex-labellings which can be used to represent the cost of opening new facilities, or edge-labellings which map the distance between adjacent cities, others describe important mathematical structures and graph-theoretical concepts.

\footnotetext{
*Partially supported by FAPESP grant 2015/11937-9, and CNPq grants 425340/2016-3 and 308689/2017-8.
} 
This thesis studied one such case. We considered a class of proper labelling problems whose goal is to assign labels to a set of elements of a graph in order to induce a vertex-colouring; the colour of a vertex is determined by the largest gap among labels assigned to its adjacent or incident elements. The many different aspects of these gap-labellings led us to investigate the problem from various points of view in Theoretical Computer Science. Our results were obtained in the form of labelling techniques, structural results for families of graphs, and both NP-completeness and polynomial-time algorithmic results.

\subsection{Notation}

Before we present the results discussed in this thesis, it is necessary to define the notation adopted in the text. Let $G$ be a simple, undirected and finite graph with vertex set $V(G)$ and edge set $E(G)$. The elements of $G$ are its vertices and its edges. The neighbourhood of a vertex $v \in V(G)$ is denoted by $N(v)$ and the set of edges incident with a vertex $v$, by $E(v)$. The degree of $v$ is denoted by $d(v)$ and, finally, the minimum and maximum degree of $G$ are denoted by $\delta(G)$ and $\Delta(G)$, respectively.

A proper (vertex-)colouring of $G$ is an assignment $c: V(G) \rightarrow \mathcal{C}$, where $\mathcal{C}$ denotes a set of colours, such that $c(u) \neq c(v)$ for every edge $u v \in E(G)$. The least number of colours for which $G$ admits such a colouring is called the chromatic number of $G$ and is denoted by $\chi(G)$. A proper labelling of $G$ is a pair $\left(\pi, c_{\pi}\right)$ where $\pi: S \rightarrow$ $\{1, \ldots, k\}$ is an assignment of numerical labels to a set $S$ of elements of $G$, and $c_{\pi}$ is a proper colouring of $G$, induced by $\pi$ through some mathematical function over the set of labelled elements. If $S=E(G)$, then $\left(\pi, c_{\pi}\right)$ is a proper edge-labelling; similarly, if $S=V(G)$, it is called a proper vertex-labelling. In this work, we considered proper labellings induced by the gaps of labels, which are defined in the following section.

\section{Proper gap-labellings}

A gap- $[k]$-edge-labelling of a graph $G$ is a proper edge-labelling of $G$ over the set $[k]=$ $\{1, \ldots, k\}$ such that $c_{\pi}: V(G) \rightarrow\{0,1, \ldots, k\}$ is a proper colouring of $G$ in which, for every vertex $v \in V(G)$, its colour $c_{\pi}(v)$ is: $\max _{e \in E(v)}\{\pi(e)\}-\min _{e \in E(v)}\{\pi(e)\}$ if $d(v) \geq 2 ; \pi(e)_{e \in E(v)}$ if $d(v)=1$; and 1, otherwise. That is, every vertex of degree at least two has its colour induced by the largest gap among the labels of its incident edges. The least $k$ for which $G$ admits a gap- $[k]$-edge-labelling is called the edge-gap number of $G$ and is denoted by $\chi_{E}^{\mathrm{g}}(G)$. Figure 1 illustrates a gap-[3]-edge-labelling of a tree.

The vertex variant of proper gap-labellings is defined similarly. A gap- $[k]$-vertexlabelling of a graph $G$ is a pair $\left(\pi, c_{\pi}\right)$ in which $\pi$ is a vertex-labelling of $G$ and $c_{\pi}$ is a proper vertex-colouring of $G$, such that: the colour of a vertex $v$, with $d(v) \geq 2$, is induced by the largest gap among the labels in $N(v)$; degree-one vertices receive as induced colour the label assigned to its neighbour; and isolated vertices receive colour 1 . Similarly to the edge version, the vertex-gap number of a graph $G$, denoted by $\chi_{V}^{\mathrm{g}}(G)$, is the least $k$ for

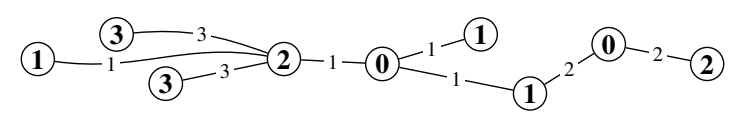

Figure 1. A gap-[3]-edge-labelling of a tree. 
which $G$ admits a gap- $[k]$-vertex-labelling. An interesting remark is that every graph with no connected component isomorphic to $K_{2}$ admits a gap-[k]-edge-labelling, for some $k \in \mathbb{N}$, whereas there are infinite families of graphs for which no gap- $[k]$-vertex-labelling exists, regardless of the number of labels.

Gap- $[k]$-edge-labellings were introduced by [Tahraoui et al. 2012] as a closing comment in their article about gap- $k$-colourings. It was, however, only formally defined by [Dehghan et al. 2013] in the following year. In this same article, the authors also introduced the vertex variant of this labelling and showed that both versions of proper gaplabellings are NP-complete when $k \geq 3$. For the particular case of $k=2$, they discuss that there is an apparent dichotomy regarding bipartite graphs, e.g. it is NP-complete to decide whether a planar bipartite graph admits a gap-[2]-edge-labelling, but if the graph is planar, bipartite and with minimum degree (at least) two, the problem can be solved in polynomial-time. The authors also question the computational complexity of deciding whether a cubic bipartite graph admits a gap-[2]-vertex-labelling, and if there exists a polynomial-time algorithm that decides whether there exists some $k \in \mathbb{N}$ for which a given graph admits a gap- $[k]$-vertex-labelling. This article, and the problems it poses, were the starting point of the work in this thesis. Our main objectives were to investigate the computational complexity aspects of proper gap-labellings of graphs and structural properties of these labellings.

In the following subsections we list the main results presented in this thesis. We begin by proving tight bounds for the edge-gap and vertex-gap numbers for some families of graphs, as well as establishing the first bounds for the vertex-gap number of arbitrary graphs. Next, we focused on proper gap-vertex-labellings and approached one of the problems posed in [Dehghan et al. 2013]. We studied the gap-[2]-vertex-labelling of cubic bipartite hamiltonian graphs, as well as the complexity of this labelling when restricted to subcubic bipartite graphs. Finally, we introduced a new parameter for graphs $G$ that do not admit any gap- $[k]$-vertex-labellings, for any $k \in \mathbb{N}$, called the gap-strength of $G$, and established bounds for this parameter for complete graphs.

\subsection{The edge-gap and vertex-gap number of graphs}

Our first goal was to better understand the intricacies and difficulties of establishing gap-labellings, in both variants of the problem. In particular, we wanted to investigate for which classes of graphs the problem remained NP-hard or, conversely, could be solved in polynomial-time. In this regard, we observed that the literature lacked results even for traditional families of graphs, e.g., there was no bound on the edge-gap and vertex-gap numbers of cycles. Therefore, we began by studying and determining these parameters for cycles and, subsequently, for increasingly more structured classes of graphs. The families we considered and the results obtained are presented in Table 1. Tightness for the edge-gap number relies on a result by [Brandt et al. 2016], which states that any given graph $G$ has $\chi_{E}^{\mathrm{g}}(G) \in\{\chi(G), \chi(G)+1\}$. As for the vertex-gap number, we proved both a lower (Theorem 3.7) and an upper bound (Theorem 4.13) for this parameter in the thesis, which yield the following result.

Corollary 5.2 (cf. [Weffort-Santos 2018]). Let $G$ be a gap-vertex-labelable graph. Then, $\chi(G) \leq \chi_{V}^{g}(G) \leq 2^{n}$, unless $G \cong K_{1, n}, n \geq 2$, in which case $\chi_{V}^{g}(G)=1=\chi(G)-1 . \square$ 


\begin{tabular}{|c|c|c|c|}
\hline Class & Edge-gap number & Vertex-gap number & Theorems \\
\hline Cycles & \multicolumn{2}{|c|}{$\chi_{E}^{\mathrm{g}}\left(C_{n}\right)=\chi_{V}^{\mathrm{g}}\left(C_{n}\right) \begin{cases}4, & \text { if } n=3 \\
2, & \text { if } n \equiv 0(\bmod 4) \\
3, & \text { otherwise }\end{cases}$} & 2.2 and 3.10 \\
\hline Crowns & \multicolumn{2}{|c|}{$\chi_{E}^{\mathrm{g}}\left(R_{n}\right)=\chi_{V}^{\mathrm{g}}\left(R_{n}\right)=\chi\left(R_{n}\right)$} & 2.3 and 3.12 \\
\hline Wheels & $\chi_{E}^{\mathrm{g}}\left(W_{n}\right)= \begin{cases}4, & \text { if } n=4 \\
\chi\left(W_{n}\right), & \text { otherwise }\end{cases}$ & $\chi_{V}^{\mathrm{g}}\left(W_{n}\right)= \begin{cases}3, & \text { if } n \geq 8 \text { and even } \\
4, & \text { if } n \geq 5 \text { and odd }\end{cases}$ & 2.4 and 3.13 \\
\hline Unicyclic graphs & $\chi_{E}^{\mathrm{g}}(G)=3$, if $p$ is odd & $\chi_{V}^{\mathrm{g}}(G)= \begin{cases}2, & \text { if } p \text { is even and } G \neq C_{n}, n \equiv 2(\bmod 4) ; \\
3, & \text { otherwise }\end{cases}$ & 2.5 and 3.15 \\
\hline $\begin{array}{l}\text { First Blanuša Snarks, Flower Snarks, } \\
\text { Goldberg Snarks and Twisted Goldberg Snarks }\end{array}$ & & $\chi_{E}^{\mathrm{g}}(G)=\chi_{V}^{\mathrm{g}}(G)=3$ & $\begin{array}{l}2.7,2.9,2.10,3.24, \\
3.26,3.27 \text { and } 3.28\end{array}$ \\
\hline Second Blanuša Snarks & open & $\chi_{V}^{\mathrm{g}}\left(B_{i}^{2}\right)=3$ & 2.8 \\
\hline
\end{tabular}

Table 1. Results for the edge-gap and vertex-gap numbers for classes of graphs. Theorem numbers reference the results in the thesis.

Up to the writing process, we were unable to determine the edge-gap number for the family of bipartite unicyclic graphs. In fact, during our work, we observed that there is an infinite family of such graphs that do not admit gap-[2]-edge-labellings. After the thesis' publication, we continued to investigate this family of graphs and devised a polynomial-time algorithm which decides whether an even-length unicyclic graph admits a gap-[2]-edge-labelling. This result was later incorporated to one of the submitted papers.

\subsection{Gap-[2]-vertex-labellings of cubic and subcubic bipartite graphs}

As previously mentioned, our main objective was to study computational complexity aspects of gap-labellings. Concerning the vertex variant, [Dehghan et al. 2013] posed the problem of determining the computational complexity of deciding whether a cubic bipartite graph admits a gap-[2]-vertex-labelling. We pursued two approaches to the problem.

The first approach was an attempt at characterizing which of these graphs admit a gap-[2]-vertex-labelling. We began by considering a restriction of this family, namely cubic bipartite that contain a Hamiltonian Cycle $H$ and whose chords are of fixed reach, i.e. every edge $e \notin E(H)$ links vertices that are at the same distance in $H$. We referred to these graphs as $\mathrm{CBH}$-graphs with homogeneous chords. For this family, we designed eight labelling techniques and algorithms which led us to establish the following theorem.

Theorem 5.2 (cf. [Weffort-Santos 2018]). Let $G$ be a CBH-graph. Then, $\chi_{V}^{g}(G)=2$ if:

(i) $n \equiv 0(\bmod 4)$; or

(ii) $n \equiv 2(\bmod 4)$ and there exists a chord $e \in E(G)$ s.t. $r(e) \equiv 3(\bmod 4)$; or

(iii) $n \equiv 2(\bmod 4)$ and $n=\beta(r+1)+\alpha(r-1)$, for $\alpha, \beta$ odd; or

(iv) $n \equiv 2(\bmod 4)$ and $r(e) \equiv 3(\bmod 6)$ for every chord $e \in E(G)$.

We also devised an Integer Linear Programming (ILP) formulation that finds a gap-[2]-vertex-labelling of a CBH-graph with homogeneous chords, if one exists. Our computational experiments and the coverage of this class provided by Theorem 5.2 led us to pose the following conjecture. 
Conjecture 3.22 (cf. [Weffort-Santos 2018]). Let $G$ be a CBH-graph not isomorphic to the Heawood Graph. Then, $\chi_{V}^{g}(G)=2$.

The second avenue of attack was an investigation on the NP-hardness aspects of gap-[2]-vertex-labellings of cubic bipartite graphs. It is known that the problem is polynomial-time solvable for bipartite graphs that are 2-regular (i.e. cycles) or $r$-regular, with $r \geq 4$. However, the boundaries of tractability are quite unclear when considering cubic (i.e. 3-regular) bipartite graphs. Therefore, in order to advance in this front, we decided to broaden our set of instances by considering subcubic bipartite graphs, which are graphs $G$ with $\Delta(G) \leq 3$. Upon such consideration, we devised a polynomial-time reduction from the MONOCHROMATIC TRIANGLE problem, which is known to be NP-complete, to our problem of deciding whether a subcubic bipartite graph admits a gap-[2]-vertex-labelling (G2VL), thus establishing the following theorem.

Theorem 3.1 (cf. [Weffort-Santos 2018]). G2VL is NP-complete, even when restricted to subcubic bipartite graphs.

Furthermore, by performing a small modification to the reduction algorithm, the same NP-hardness result holds even when the graph is subcubic with $\delta(G) \geq 2$. This result contributes significantly to narrowing down the boundaries of tractability for the G2VL problem.

\subsection{The gap-strength of complete graphs}

Our final considerations are on another perspective on gap- $[k]$-vertex-labellings. As previously mentioned, there are graphs for which no gap- $[k]$-vertex-labellings exist, regardless of the value of $k$. We proved that this is the case for the family of complete graphs $K_{n}$, $n \geq 4$, and for a subfamily of split graphs. In fact, these results came upon addressing the problem of determining the computational complexity of deciding whether a graph $G$ is gap-vertex-labelable, also posed by [Dehghan et al. 2013].

Since no upper bound was previously known for the vertex-gap number of graphs prior to our work, our first result was to determine that every gap-vertex-labelable graph admits a labelling using only distinct powers of two. This, in turn, implies that there exists a $\mathcal{O}(n$ !)-time algorithm which decides this problem — one need only verify every possible assignment of different powers of two to the vertices of the graph.

However, another question arose upon our considerations of Dehghan et al.'s problem: what is the least number of edges one must remove from a complete graph such that the resulting graph is gap-vertex-labelable? This problem led us to define and study a new parameter $\operatorname{str}_{\text {gap }}(G)$, which we coined the gap-strength of a (nongap-vertex-labelable) graph $G$. We investigated this value for complete graphs from a structural point of view, establishing that $\operatorname{str}_{\text {gap }}\left(K_{n}\right) \in \mathcal{O}(n \sqrt{n})$. We also approached the parameter from an algorithmic perspective, designing a dynamic programming algorithm which computes a lower bound on the gap-strength of $K_{n}$. The experimental results show that $n \sqrt{n}$ also appears to be a lower bound for $\operatorname{str}_{\text {gap }}\left(K_{n}\right)$. This led us to pose the following conjecture.

Conjecture 5.3 (cf. [Weffort-Santos 2018]). Let $K_{n}$ be the complete graph of order $n$. Then, $\operatorname{str}_{\text {gap }}\left(K_{n}\right) \in \Theta(n \sqrt{n})$. 


\section{Concluding remarks}

This thesis presents a comprehensive study of proper gap-labellings of graphs. It establishes the edge-gap and vertex-gap numbers for several families of graphs, NP-hardness proofs, different labelling techniques, ILP formulations and dynamic programming algorithms, as well as the introduction, investigation and bounds on a new parameter. The results in this thesis were submitted to conferences in the areas of Graph Theory, Combinatorics and Theoretical Computer Science. The list of currently published works follows:

- [Weffort-Santos et al. 2017] Extended abstract II ETC'2017: presents the tight bounds for the edge-gap and vertex-gap numbers of cycles, crowns and wheels.

- [Weffort-Santos et al. 2018] Abstract VIII LAWCG'2018: contains our results on the vertex-gap number of unicyclic graphs, as well as for the edge-gap number of odd-length unicyclic graphs; the polynomial-time algorithm mentioned in Section 2.1 was obtained after the time of publication.

- [Weffort-Santos et al. 2019b] Extended abstract Special Issue of Matemática Contemporânea for the LAWCG'2018 conference (to appear).

- [Weffort-Santos et al. 2019a] Extended abstract X LAGOS'2019 (to appear): presents the proof of Theorem 2.2 and discussions on the problem.

In addition, there are three full papers in preparation, which will be submitted to specialized journals until the end of the semester. The first contains our results on the edge-gap and vertex-gap numbers of snarks. The second contains our results for $\mathrm{CBH}-$ graphs and on the NP-hardness on subcubic bipartite graphs; this paper will be submitted to the special issue of Discrete Applied Mathematics for the LAGOS'2019 conference. The last paper comprises our results on the gap-strength of complete graphs.

\section{References}

Brandt, A., Moran, B., Nepal, K., Pfender, F., and Sigler, D. (2016). Local gap colorings from edge labelings. Australasian Journal of Combinatorics, 65(3):200 - 211.

Dehghan, A., Sadeghi, M., and Ahadi, A. (2013). Algorithmic complexity of proper labeling problems. Theoretical Computer Science, 495:25 - 36.

Tahraoui, M. A., Duchêne, E., and Kheddouci, H. (2012). Gap vertex-distinguishing edge colorings of graphs. Discrete Mathematics, 312(20):3011 - 3025.

Weffort-Santos, C. A. (2018). Proper gap-labellings: on the edge and vertex variants. Master's thesis, University of Campinas.

Weffort-Santos, C. A., Campos, C. N., and Schouery, R. C. S. (2017). Tight bounds for gap-labellings. In Anais do XXXVII Congresso da Sociedade Brasileira de Computação, pages 119-122.

Weffort-Santos, C. A., Campos, C. N., and Schouery, R. C. S. (2018). Proper gaplabellings of unicyclic graphs. In Anais do VIII Latin American Workshop on Cliques in Graphs, page 34.

Weffort-Santos, C. A., Campos, C. N., and Schouery, R. C. S. (2019a). On the complexity of gap-[2]-vertex-labellings of subcubic bipartite graphs. Accepted in X LAGOS'2019.

Weffort-Santos, C. A., Campos, C. N., and Schouery, R. C. S. (2019b). Proper gaplabellings of unicyclic graphs. Accepted in the Special Issue of Matemática Contemporânea for the LAWCG'2018 conference. 